\title{
Transcellular Model for Neutral and Charged Nanoparticles Across an In Vitro Blood-Brain Barrier
}

\author{
Lin Zhang, Jie Fan, Guanglei Li, Zhaokai Yin, and Bingmei M. Fu (D) \\ Department of Biomedical Engineering, The City College of the City University of New York, 160 Convent Ave, New York, \\ NY 10031, USA
}

(Received 25 June 2020; accepted 15 October 2020; published online 28 October 2020)

Associate Editor Hanjoong Jo oversaw the review of this article.

\begin{abstract}
Purpose-The therapeutic drug-loaded nanoparticles (NPs, 20-100 $\mathrm{nm}$ ) have been widely used to treat brain disorders. To improve systemic brain delivery efficacy of these NPs, it is necessary to quantify their transport parameters across the blood-brain barrier (BBB) and understand the underlying transport mechanism.

Methods-Permeability of an in vitro BBB, bEnd3 (mouse brain microvascular endothelial cells) monolayer, to three neutral NPs with the representative diameters was measured using an automated fluorometer system. To elucidate the transport mechanism of the neutral NPs across the in vitro $\mathrm{BBB}$, and that of positively charged NPs whose BBB permeability was measured in a previous study, we developed a novel transcellular model, which incorporates the charge of the in vitro $\mathrm{BBB}$, the mechanical property of the cell membrane, the ion concentrations of the surrounding salt solution and the size and charge of the NPs.

Results - Our model indicates that the negative charge of the surface glycocalyx and basement membrane of the BBB plays a pivotal role in the transcelluar transport of NPs with diameter 20-100 nm across the BBB. The electrostatic force between the negative charge at the in vitro $\mathrm{BBB}$ and the positive charge at NPs greatly enhances NP permeability. The predictions from our transcellular model fit very well with the measured BBB permeability for both neutral and charged NPs.

Conclusion - Our model can be used to predict the optimal size and charge of the NPs and the optimal charge of the BBB for an optimal systemic drug delivery strategy to the brain.
\end{abstract}

Keywords-Transcellular model, Blood-brain barrier, Nanoparticle, Charge, Permeability, bEnd3 (mouse brain microvascular endothelial cells).

Address correspondence to Bingmei M. Fu, Department of Biomedical Engineering, The City College of the City University of New York, 160 Convent Ave, New York, NY 10031, USA. Electronic mail: fu@ccny.cuny.edu

\section{INTRODUCTION}

The blood-brain barrier (BBB) is an interface between central nervous system (CNS) and blood circulation. It limits exchange of substances between blood circulation and brain tissue to prevent the CNS from the blood-borne toxins but it hinders the systemic delivery of therapeutic drugs to the brain. ${ }^{1,14}$ Over the last two decades, researchers have developed many methods to improve the brain drug delivery, one of which is to use nanoparticles (NPs) as drug carriers for drug delivery. ${ }^{6,9,12,32,39}$ because NPs can be made nontoxic, biodegradable and biocompatible. They can also maintain physical stability in the blood and have relatively long blood circulation time. Most importantly, after being conjugated with specific antibodies or peptides, they have the ability to recognize targeting sites. ${ }^{28}$ NPs, made of liposomes, polymers and metals, usually have diameters from 10 to $1000 \mathrm{~nm}$. It has been found that NPs under $200 \mathrm{~nm}$ in diameter display a decreased rate of plasma clearance and thus an extended circulation time as compared to those with a larger diameter. ${ }^{32,37}$

There are two transport pathways across the BBB, the paracellular and transcellular pathways. ${ }^{14,29}$ Water and small hydrophilic solutes cross the BBB through the paracellular pathway, and the large molecules cross the BBB through transcellular pathways. ${ }^{14,20,25,32,39}$ Previous studies have investigated the transport across the $\mathrm{BBB}$ both in vivo and in vitro. By employing intravital and multiphoton microscopy, Yuan ${ }^{45,46}$ and Shi et al. ${ }^{35,36}$ developed a non-invasive method and quantified the permeability $(\mathrm{P})$ of post-capillary venules either on rat pia mater or in brain parenchyma $100-200 \mu \mathrm{m}$ below pia mater to various sized solutes 
and NPs. Meanwhile, in vitro BBB models by monoculturing or co-culturing brain microvascular endothelial cells (ECs) with astrocytes and/or pericytes ${ }^{14,24}$ were developed for low cost, high-throughput screening, and easiness to assess compounds as well as to investigate the transport mechanism at molecular levels. These studies showed that the endothelial monoculture and the co-culture models are fairly good models for studying the transport of relatively large solutes across the BBB.

Yuan et al. $^{44}$ measured the permeability of an in vitro $\mathrm{BBB}$ formed by bEnd3 (mouse brain microvascular ECs) monolayer to charged solutes and NPs. They found that at the surface of bEnd 3 monolayer there is a glycocalyx layer with comparable thickness and charge density as observed in cerebral and peripheral microvessels. ${ }^{3,15,42}$ They also found that the permeability of the bEnd 3 monolayer to positively charged NPs of diameter $66-88 \mathrm{~nm}$ is the same as that to a much smaller solute, Dextran $4 \mathrm{k}$ (Stokes diameter $\sim 2.8 \mathrm{~nm}$ ). Since the width of the paracellular pathway between ECs is only $\sim 18 \mathrm{~nm}$ or less, ${ }^{23,25}$ the 66-88 nm diameter NPs are most likely across the bEnd3 monolayer through a transcellular or transcytosis pathway. The transcytosis induced by the electrostatic effect have been also studied with cationized solutes, such as charged gold NPs and latex NPs. ${ }^{4,17,19,38,41,44}$

In this study, we measured the permeability of an in vitro $\mathrm{BBB}$ (bEnd 3 monolayer) to neutral NPs from 20 to $100 \mathrm{~nm}$ in diameter. The permeability of these neutral NPs is $\sim 100$ times smaller comparing with that of the positively charged NPs with the size from 66 to $88 \mathrm{~nm}^{44,45}$ From these experimental data, we hypothesized that the NP, which is larger than the width of the inter-endothelial cleft, crosses the BBB by transcytosis, and the charge carried by the NP enhances the rate of this process. To test these hypotheses and to quantitatively investigate the transcytosis mechanism by which a NP crosses the in vitro $\mathrm{BBB}$ (bEnd3 monolayer), we developed a transcellular model for the NP transport across brain microvascular ECs.

As summarized in Zhang et al. ${ }^{48}$ and Hui et al., ${ }^{21}$ the physiochemical and mechanical properties of the cell membrane and those of the NP, the size and shape of the NP, as well as the local environment of cells, all of which affect the transcytosis of a NP. A recent review by Pulgar ${ }^{31}$ indicates that transcytosis across the $\mathrm{BBB}$ can be receptor-mediated or charge dependent. Gao et al. ${ }^{16,43}$ developed a model for receptor-mediated endocytosis. Fleck and Netz ${ }^{13}$ developed a model to describe the wrapping process of a charged mem- brane and an oppositely charged NP. They investigated the effects of ion concentrations of the surrounding salt solution and physical properties of cell membrane on the wrapping process. Based on their wrapping model, we further assumed a standard linear solid model for the viscoelastic property of the EC membrane, ${ }^{26,33,34}$ and developed a new transcellular model for a NP across the in vitro BBB (bEnd3 monolayer). Our model incorporated the charge of bEnd3 monolayer, the viscoelastic property, bending rigidity and surface tension of the EC membrane, the ion concentrations of the surrounding salt solution, as well as the size and charge of the NPs. Our model revealed that the negative charge of the surface glycocalyx and basement membrane of the BBB plays a pivotal role in the transcelluar transport of NPs with diameter $20-100 \mathrm{~nm}$. The electrostatic force between the negative charge at the in vitro $\mathrm{BBB}$ and the positive charge at the NP greatly increases NP permeability. The predictions from our model reconcile with the measured BBB permeability for both neutral and charged NPs. Our model can be further employed to develop optimal systemic drug delivery strategy to the brain by predicting the optimal size and charge of the NPs, as well as the optimal charge of the BBB.

\section{MATERIALS AND METHODS}

\section{Determination of In Vitro BBB Permeability to Nanoparticles (NPS)}

\section{Materials}

Fluorescent polystyrene nanospheres with representative diameters 22,48 and $100 \mathrm{~nm}$ were purchased from the Fisher Scientific (Waltham, MA). Fibronectin and bovine serum albumin (BSA) were purchased from Sigma-Aldrich (St. Louis, MO). Fetal bovine serum (FBS) was from Hyclone (Logan, UT). Immortalized mouse cerebral microvascular endothelial cells (bEnd3) were from American Type Culture Collection (ATCC, Manassas, VA). Transwell filters $(0.4 \mu \mathrm{m}$ pore size, $12 \mathrm{~mm}$ diameter, 12 inserts in a plate) were purchased from Corning Inc. (Corning, NY).

\section{Cell Culture}

The detailed method for generating in vitro BBB model was described in Refs. ${ }^{10,11,24,44}$. Briefly, bEnd3 cells were grown in DMEM/F-12 (Dulbecco's Modified Eagle's Medium/nutrient mixture F-12 Ham) with $10 \% \mathrm{FBS}, 2 \mathrm{mM}$ L-glutamine, $50 \mathrm{U} / \mathrm{mL}$ penicillin and $50 \mu \mathrm{g} / \mathrm{mL}$ streptomycin. Cells were maintained in a humidified cell culture incubator with $5 \% \mathrm{CO}_{2}$ at 
$37^{\circ} \mathrm{C}$. Cells used in the experiment were seeded at $6.0 \times 10^{4}$ cells $/ \mathrm{cm}^{2}$ on the Transwell permeable filters. The filters were first incubated at $37{ }^{\circ} \mathrm{C}$ with $200 \mu \mathrm{L}$ of $30 \mu \mathrm{g} / \mathrm{mL}$ fibronectin for $1.5 \mathrm{~h}$ before seeding the cells. The cells seeded onto the Transwell filters reached confluence in 3-4 days. The permeability experiments were performed on the monolayer 4-5 days after cell seeding, allowing sufficient time for the cultured cells to develop the junctions between cells.

\section{Permeability Measurement}

The permeability $(P)$ of the in vitro BBB to NPs was measured using an automated fluorometer system. ${ }^{7,8,24}$ On the day of the experiment, the Transwell insert filter with the cell monolayer was washed twice with the experimental solution containing $1 \%$ BSA and $1 \%$ FBS in DMEM medium free of phenol red, and then was sealed within a transport chamber to form upper and bottom compartments. The upper compartment was filled with $1 \mathrm{~mL} 0.025 \mathrm{nM}$ polystyrene NPs in the experiment solution, while the bottom compartment was filled with the experimental solution only. The bottom compartment was connected to a laser excitation source and an emission detector via optical fibers. The fluorescent NPs in the bottom compartment were excited by the excitation light produced by a $10-\mathrm{mW}$ Crystal laser and the emission was counted by a photon counting detector. The data was recorded by the FluoroMeasure acquisition software (C\&L Instruments, Hummelstown, PA). Both the upper and bottom compartments were continuously supplied with $5 \% \mathrm{CO}_{2}$ to maintain the $\mathrm{pH}$ of the medium at 7.4. The temperature of the whole permeability measurement system was maintained at $37^{\circ} \mathrm{C}$.

FluoroMeasure acquisition software was used to measure the fluorescence intensity in the bottom compartment, which was recorded every $10 \mathrm{~s}$ for the duration of the measurement $(2 \mathrm{~h})$. The intensity was converted to the concentration by a calibration curve, and the permeability $\mathrm{P}$ of the monolayer to NPs was calculated by

$$
P=\frac{V_{b}}{A} \frac{\Delta C_{b} / \Delta t}{C_{u}}
$$

where $P$ is the permeability, $\Delta C_{\mathrm{b}} / \Delta t$ is the increase rate of the NP concentration in the bottom compartment, calculated through the slope of the intensity vs. time curve, which was directly measured in the experiment. $V_{\mathrm{b}}$ is the fluid volume in the bottom compartment, $C_{\mathrm{u}}$ is the NP concentration in the upper compartment, which was assumed to be constant due to low permeability of the cell monolayer. $A$ is the surface area of the porous membrane of the Transwell insert filter.

\section{Transcellular Model Formation}

\section{Model Geometry}

Figure 1 shows the model geometry. Since NPs (20$100 \mathrm{~nm}$ ) under study are much smaller than the endothelial cell (EC, $\sim 20 \mu \mathrm{m}$ ), the EC membrane was assumed to be infinite compared to the NP and was described as a two-dimensional membrane with the bending rigidity $\mathrm{kc}$ and the surface tension $\gamma^{5,49}$ Due to a very dilute solution of NPs, the relative ratio to the water concentration is $4.5 \times 10^{-13}$, the interactions between NPs were negligible. The unwrapped region of the EC membrane was assumed to be flat. The negative charge at the luminal surface of the EC is due to the surface glycocalyx $\mathrm{x}^{2,11,44}$ and that at the abluminal side is due to the extracellular matrix. ${ }^{11,23}$ The charge densities $\sigma$ at the luminal and abluminal sides of the EC were assumed to be the same $\mathrm{e}^{23}$ and uniform along the EC surface. The charge density $\rho$ at the NP was also assumed to be uniform along the NP surface. We considered neutral and positively charged NPs in the present study. The NP crosses the EC membrane by endocytosis and exocytosis due to the electrostatic force as well as the mechanical property of the EC membrane, while it crosses the EC cytoplasm by diffusion.

\section{Free energy}

For the interaction of an oppositely charged sphere and the cell membrane in a salt solution, a total energy can be expressed as the following, in the unit of $K_{\mathrm{B}} T$. $^{13}$ Here $K_{\mathrm{B}}$ is the Boltzmann constant and $T$ is the absolute temperature.

$$
\begin{gathered}
E=E_{\text {mech }}+E_{\text {attr }}+E_{\text {rep }} \\
E_{\text {mech }}=\frac{K_{C}}{2} \int d^{2} n \sqrt{g(n)}(\Delta X(n))^{2}+\gamma_{\mathrm{eff}} \int d^{2} n \sqrt{g(n)} \\
E_{\mathrm{attr}}=-\frac{l_{B} 4 \pi R^{2} \rho \sigma e^{k R}}{1+\kappa R} \int d^{2} n \sqrt{g(n)} \frac{\exp [-k|X(n)|]}{|X(n)|} \\
E_{\text {rep }}=-\frac{l_{B} \sigma^{2}}{2} \int d^{2} n \int d^{2} n^{\prime} \sqrt{g(n) g\left(n^{\prime}\right)} \frac{\exp \left[-k\left|X(n)-X\left(n^{\prime}\right)\right|\right]}{\left|X(n)-X\left(n^{\prime}\right)\right|}
\end{gathered}
$$

Here $E_{\text {mech }}$ is the energy due to the mechanical deformation of the cell membrane. The first term in $\mathrm{E}_{\text {mech }}$ is due to the bending and the second term due to the surface tension. $E_{a t t r}$ is the energy due to the attraction between an oppositely charged sphere and the cell membrane. $\mathrm{E}_{\mathrm{rep}}$ is the energy due to the 
(a)
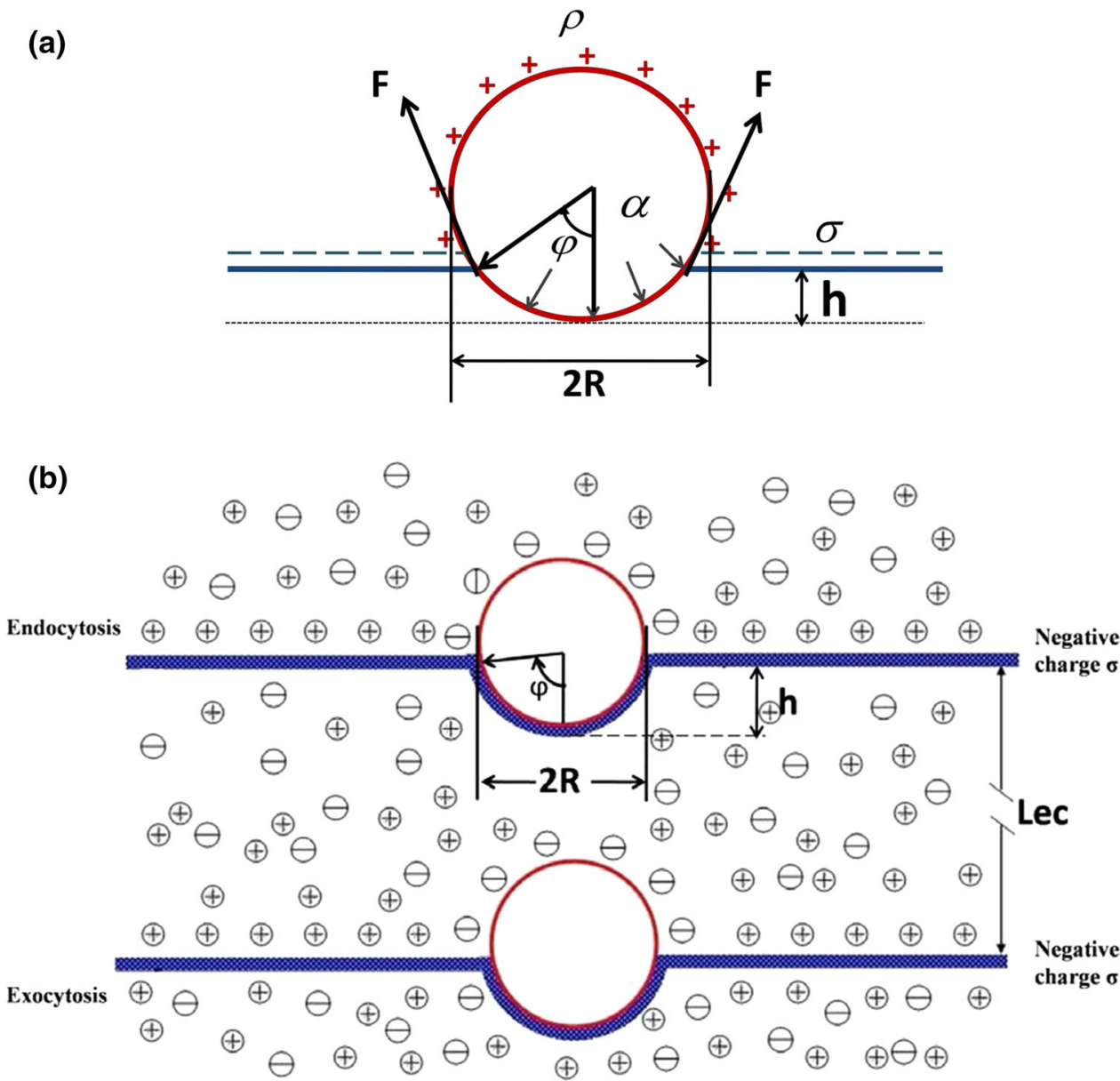

FIGURE 1. Model geometry for the transcellular transport of a NP across the in vitro BBB (not in scale). (a) a NP of diameter 2R and charge density $\rho$ penetrating a cell membrane with charge density $\sigma . h$ is the penetration depth of the NP. F is the force due to interaction of the NP and the cell membrane and $\alpha$ is the stress perpendicular to the interface between the NP and the cell membrane. (b) Illustration for transcellular transport of a NP across an EC: endocytosis of the NP into an EC from the luminal surface, diffusion of the NP in the cytoplasm of the EC with thickness Lec, and exocytosis of the NP from the abluminal surface of the EC.

repulsion between one part of the cell membrane and another part of the cell membrane. $\mathrm{X}(\mathbf{n})$ is the position on the membrane, $\mathbf{n}=\left(\mathrm{n}_{1}, \mathrm{n}_{2}\right)$ is the internal coordinates of the membrane, $\mathrm{g}(\mathbf{n})$ is the determinant of the induced metric field defined by $g_{i, j}=\partial_{i} X \partial_{j} X$ and $\Delta=$ $g^{-\frac{1}{2}} \partial_{i}\left(\sqrt{g} g_{i, j} \partial_{j}\right) \cdot \gamma_{\text {eff }}$ is the effective surface tension $\gamma_{\mathrm{eff}}=$ $\gamma-\gamma_{\text {ele }}$ where $\gamma$ is the mechanical surface tension of the cell membrane and $\gamma_{\text {ele }}$ is the electrostatic surface tension of the cell membrane due to its charge and its surrounding salt solution $\gamma_{\text {ele }}=\pi l_{B} \sigma^{2} / k$. Here $1 / \mathrm{k}$ is the Debye-Hückle length, $k=\sqrt{8 \pi l_{\mathrm{B}} \mathrm{C}}$, in which $\mathrm{C}$ is the ion concentration of the surrounding solution. $l_{\mathrm{B}}$ is the Bjerrum length, $l_{\mathrm{B}}=\frac{e^{2}}{4 \pi \varepsilon_{0} \varepsilon_{\mathrm{r}} K_{\mathrm{B}} T}$, which measures the distance at which two elementary charges interact with the thermal energy $K_{\mathrm{B}} T^{33} \cdot \varepsilon_{0}, \varepsilon_{\mathrm{r}}$ are the vacuum permittivity and relative permittivity of the surrounding solution, respectively.
In the spherical coordinate, the above equations can be simplified and rescaled into dimensionless forms with the characteristic parameters ${ }^{13}: \tilde{E}=E / 2 \pi k_{c}$, $\tilde{Z}=\rho / \sigma, \quad \tilde{\sigma}=\sigma \sqrt{l_{\mathrm{B}} R^{3} / K_{\mathrm{c}}}, \quad \tilde{\gamma}_{\mathrm{eff}}=\gamma_{\mathrm{eff}} R^{2} / K_{\mathrm{c}} . \quad$ Here, $K_{\mathrm{C}}=k_{\mathrm{c}} / K_{\mathrm{B}} T, k_{\mathrm{c}}$ is the bending rigidity of the cell membrane, $\sigma$ is the charge density of the EC membrane, $\rho$ is the charge density of a NP, and R is the NP radius. $h$ is the penetrating depth for the wrapping (Fig. 1), $\tilde{h}=h / R=1-\cos \varphi$. When $\sqrt{\tilde{h}}>\sqrt{2} / \kappa R$, $k R \gg 1$ as in our case $(k R>25)$, we have,

$$
\begin{aligned}
& \tilde{E}_{\text {mech }}=2 \tilde{h}+\tilde{\gamma}_{\text {eff }} \tilde{h}, \\
& \tilde{E}_{\text {attr }}=-\frac{4 \pi \tilde{\sigma}^{2} \tilde{Z}}{1+k R} \tilde{h},
\end{aligned}
$$




$$
\begin{aligned}
\tilde{E}_{\text {rep }}= & \frac{2 \pi \tilde{\sigma}^{2}}{(k R)^{2}}-\frac{\sqrt{2} \tilde{\sigma}^{2}}{(k R)^{2}} \tilde{h}^{1 / 2}+\frac{\tilde{\sigma}^{2}}{4 \sqrt{2}(k R)^{2}} \tilde{h}^{3 / 2} \\
& +\frac{\tilde{\sigma}^{2}}{16 \sqrt{2}(k R)^{2}} \tilde{h}^{5 / 2}
\end{aligned}
$$

The higher order terms for $(\mathrm{kR})^{-3}$ and above are neglected in Eq. 7. The total energy is,

$$
\tilde{E}=\tilde{E}_{\text {mech }}+\tilde{E}_{\text {attr }}+\tilde{E}_{\text {rep }}
$$

\section{Force and Stress}

The force $\mathbf{F}$ acting on the cell membrane is the gradient of the energy and in the spherical coordinates,

$$
\mathbf{F}=-\nabla E=-\left(\overrightarrow{e_{r}} \frac{\partial E}{\partial r}+\frac{1}{r} \overrightarrow{e_{\varphi}} \frac{\partial E}{\partial \varphi}+\frac{1}{r \sin \varphi} \overrightarrow{e_{\theta}} \frac{\partial E}{\partial \theta}\right)
$$

Because $\mathbf{F}$ only acts on the interface of the NP and the cell membrane (Fig. 1a) and the material properties are uniform along the cell membrane, after putting Eqs. 5-8 into Eq. 9, Eq. 9 becomes,

$$
\begin{aligned}
\mathbf{F}= & -\frac{1}{\mathrm{R}} \mathrm{e} \frac{\partial \mathrm{E}}{\partial \varphi}=-\frac{2 \pi K_{C} \sin \varphi}{R}\left(2+\tilde{\gamma}_{\mathrm{eff}}-\frac{4 \pi \tilde{\sigma}^{2} \tilde{Z}}{1+k R}\right) \\
- & \frac{2 \pi K_{C} \sin \varphi}{R} \\
& \times\left[-\frac{\sqrt{2} \tilde{\sigma}^{2}}{2(k R)^{2}}(1-\cos \varphi)^{-\frac{1}{2}}+\frac{3 \tilde{\sigma}^{2}}{8 \sqrt{2}(k R)^{2}}(1-\cos \varphi)^{\frac{1}{2}}\right. \\
& \left.+\frac{5 \tilde{\sigma}^{2}}{32 \sqrt{2}(k R)^{2}}(1-\cos \varphi)^{\frac{3}{2}}\right]
\end{aligned}
$$

As shown in Fig. 1a, the stress acting on the interface of a NP and the cell membrane is,

$$
\alpha=\mathbf{F} / \pi R^{2} \sin \varphi
$$

when putting Eq. 10 into Eq. 11, Eq. 11 becomes,

$$
\begin{aligned}
\alpha= & -\frac{2 k_{C}}{R^{3}}\left(2+\tilde{\gamma}_{\mathrm{eff}}-\frac{4 \pi \tilde{\sigma}^{2} \tilde{Z}}{1+k R}\right)-\frac{2 k_{C}}{R^{3}} \\
& \times\left[-\frac{\sqrt{2} \tilde{\sigma}^{2}}{2(k R)^{2}}(1-\cos \varphi)^{-\frac{1}{2}}+\frac{3 \tilde{\sigma}^{2}}{8 \sqrt{2}(k R)^{2}}(1-\cos \varphi)^{\frac{1}{2}}\right. \\
& \left.+\frac{5 \tilde{\sigma}^{2}}{32 \sqrt{2}(k R)^{2}}(1-\cos \varphi)^{\frac{3}{2}}\right]
\end{aligned}
$$

The strain is defined as $\varepsilon=\left(A_{\mathrm{t}}-A_{\mathrm{o}}\right) / A_{\mathrm{o}}$, where $A_{\mathrm{t}}-A_{\mathrm{o}}$ is the change of the contacting area of the NP and cell membrane, and $A_{0}$ is the contacting area of the cell membrane before it is deformed during NP penetration. $A_{\mathrm{t}}=2 \pi R^{2}(1-\cos \varphi)$ is the contacting area of the deformed cell membrane, while,

$$
\begin{aligned}
& A_{0}=\pi(R \sin \varphi)^{2}, \text { when } \varphi \in[0, \pi / 2] \\
& A_{0}=2 \pi R^{2}, \text { when } \varphi \in[\pi / 2, \pi]
\end{aligned}
$$

then

$$
\begin{aligned}
& \varepsilon=\frac{1-\cos \varphi}{1+\cos \varphi}, \text { when } \varphi \in[0, \pi / 2] \\
& \varepsilon=-\cos \varphi, \text { when } \varphi \in[\pi / 2, \pi]
\end{aligned}
$$

\section{Constitutive Equation for the Cell Membrane}

Based on the experimental measurements, a standard linear solid (SLS) model was used to describe the viscoelastic behavior of the endothelial cell membrane. ${ }^{2,33,34}$ The constitutive equation for the standard linear solid model is

$$
\left(k_{1}+k_{2}\right) \frac{d \varepsilon}{d t}=\frac{k_{2}}{\mu} \alpha+\frac{d \alpha}{d t}-\frac{k_{1} k_{2}}{\mu} \varepsilon
$$

here $k_{1}, k_{2}$ are the spring constants in the model, which describe the elastic properties of the cell membrane, and the $\mu$ is the viscosity of the cell membrane. If $T=t / \tau$ is the dimensionless time, where

$$
\tau=\frac{k_{1}+k_{2}}{k_{1} k_{2}} \mu \text {. }
$$

Substituting Eqs. 12, 13 and 15. into Eq. 14, we obtained a non-linear first order ordinary differential equation. When $\varphi \in[0, \pi / 2]$,

$$
\frac{d \varphi}{d T}=\frac{\frac{\alpha}{k_{1}}-\frac{1-\cos \varphi}{1+\cos \varphi}}{2 \sin \varphi /(1+\cos \varphi)^{2}+\frac{1}{k_{1}+k_{2}} \frac{2 K_{c}}{R^{3}} \sin \varphi\left[\frac{1}{4} \frac{\sqrt{2} \tilde{\sigma}^{2}}{(\kappa R)^{2}}(1-\cos \varphi)^{-3 / 2}+\frac{3}{4} \frac{\tilde{\sigma}^{2}}{4 \sqrt{2}(\kappa R)^{2}}(1-\cos \varphi)^{-1 / 2}+\frac{15}{4} \frac{\tilde{\sigma} 2}{16 \sqrt{2}(\kappa R)^{2}}(1-\cos \varphi)^{1 / 2}\right]}
$$


when $\varphi \in[\pi / 2, \pi]$

$$
\frac{d \varphi}{d T}=\frac{\frac{\alpha}{k_{1}}+\cos \varphi}{\sin \varphi+\frac{1}{k_{1}+k_{2}} \frac{2 K_{c}}{R^{3}} \sin \varphi\left[\frac{1}{4} \frac{\sqrt{2} \tilde{\sigma}^{2}}{(\kappa R)^{2}}(1-\cos \varphi)^{-3 / 2}+\frac{3}{4} \frac{\tilde{\sigma}^{2}}{4 \sqrt{2}(\kappa R)^{2}}(1-\cos \varphi)^{-1 / 2}+\frac{15}{4} \frac{\tilde{\sigma} 2}{16 \sqrt{2}(\kappa R)^{2}}(1-\cos \varphi)^{1 / 2}\right]}
$$

The above equation was solved numerically by 4th order Runge-Kutta method using ode45 solver in MATLAB R2017a.

\section{Initial Condition}

For the SLS model, the springs on both arms response immediately when the force is applied and the dashpot remains still. Therefore, at the beginning when $t=0$, there is an initial penetrating distance $h(0)$ or $\varphi(0)$ due to the initial applied stress. The following equation can be used to solve for the initial $\varphi(0)$, which is the initial condition for solving Eq. 16.

$$
\alpha(\varphi(0))=\left(k_{1}+k_{2}\right) \frac{1-\cos \varphi(0)}{1+\cos \varphi(0)}
$$

here $k_{1}, k_{2}$ are the spring constants in the model, $\alpha$ is shown in Eq. 12.

\section{The BBB Permeability $(P)$ to a NP by Transcellular Transport}

Because the size of the NP under study is larger than the width of the cleft between adjacent ECs (less than $10 \mathrm{~nm}$ in the narrow region ${ }^{24}$ ), the NP can only cross the in vitro $\mathrm{BBB}$ through the transcellular pathway. At the luminal side of the EC, a NP enters into the cell by endocytosis, diffuses in intracellular compartment, and exits the cell from the abluminal side by exocytosis, as shown in Fig. 1b. The P for a NP via a transcellular pathway can be defined as,

$$
\frac{1}{P}=\frac{1}{P_{\mathrm{en}}}+\frac{1}{P_{\mathrm{ex}}}+\frac{1}{P_{\mathrm{d}}}
$$

TABLE 1. Measured permeability $(P)$ of the in vitro BBB to neutral NPs.

\begin{tabular}{ll}
\hline NP diameter $(\mathrm{nm})$ & Permeability $\pm \mathrm{SE}\left(\times 10^{-8} \mathrm{~cm} / \mathrm{s}\right)$ \\
\hline 22 & $2.6( \pm 0.7, n=8)$ \\
48 & $2.3( \pm 0.8, n=6)$ \\
100 & $2.4( \pm 0.9, n=11)$ \\
\hline
\end{tabular}

The reverse of the permeability reflects the resistance. The first two terms on the right are the resistances during endocytosis and exocytosis, respectively. The third term is the diffusive resistance when a NP moves across the intracellular compartment, assuming by diffusion, which is,

$$
P_{d}=D_{\text {eff }} / L_{\mathrm{ec}}
$$

Here $L_{\mathrm{ec}}$ is the thickness of the EC (see Fig. 1b). $D_{\text {eff }}$ is the diffusion coefficient of a NP in the cell cytoplasm, which was estimated by using the Stoke-Einstein equation,

$$
D_{\text {eff }}=\frac{K_{B} T_{t}}{6 \pi \eta R}
$$

Here $K_{\mathrm{B}}$ is the Boltzmann constant and $T_{\mathrm{t}}$ is the absolute temperature, $\eta$ is the viscosity of the cell cytoplasm, and $\mathrm{R}$ is the radius of the NP. $P_{\mathrm{en}}$ and $P_{\mathrm{ex}}$ are the NP permeability during endocytosis and the exocytosis, which were assumed to be the same and estimated as,

$$
P_{\mathrm{en}}=P_{\mathrm{ex}}=2 R / t^{*}
$$

Here $t^{*}$ is the penetrating time, which is defined as the time it takes for the cell membrane to completely engulf a NP of diameter $2 \mathrm{R}$ into the cell interior, when $h=2 \mathrm{R}$ (Figs. 1a and 1b).

\section{RESULTS}

\section{Permeability of In Vitro BBB to Neural NPS}

Table 1 shows the measured permeability of the in vitro $\mathrm{BBB}(\mathrm{P})$ to neutral NPs with diameters ranging from 22 to $100 \mathrm{~nm}$. P of all NPs is in the order of $10^{-8}$ $\mathrm{cm} / \mathrm{s}$. Statistical analysis (Sigma Plot 11.2 from Systat Software Inc., San Jose, CA) showed that the P of the $\mathrm{BBB}$ to these NPs is not significantly different $(p>0.1)$ although these NPs vary largely in size. The results imply that the NPs in this size range cross the BBB through a transcellular pathway. For these sized NPs, the resistance of diffusion in the EC cytoplasm, $L_{\mathrm{ec}} / D_{\mathrm{eff}}$, is in the order of $10^{4} \mathrm{~s} / \mathrm{cm}$ if $\mathrm{L}_{\mathrm{ec}}=2 \mu \mathrm{m} .{ }^{11} \mathrm{It}$ 
TABLE 2. Parameters used in the transcellular model.

\begin{tabular}{llll}
\hline Symbol & \multicolumn{1}{c}{ Definition } & \multicolumn{1}{c}{ Value } & Unit \\
\hline$C$ & Salt concentration & 140 & $\mathrm{mM}$ \\
$e$ & Electron charge & $1.602 \times 10^{-19}$ & $\mathrm{C}$ \\
$K_{\mathrm{B}}$ & Boltzmann constant & $1.38 \times 10^{-23}$ & $\mathrm{~J} / \mathrm{K}$ \\
$T_{\mathrm{t}}$ & Cell culture temperature & 310 & $\mathrm{~K}$ \\
$k_{\mathrm{C}}$ & Bending rigidity & $-20 \mathrm{~K}_{\mathrm{B}} \mathrm{T}^{14}$ & $\mathrm{~J}$ \\
$R$ & Radius of nanoparticles & $10-100$ & $\mathrm{~nm}$ \\
$\rho$ & charge density on nanoparticles & $0.05-0.2$ & number of positive charge/nm ${ }^{2}$ \\
$\sigma_{\mathrm{v}}$ & ESG/BM volume charge density & $20-30^{1}$ & $\mathrm{mEq} / \mathrm{L}$ \\
$\sigma$ & Area charge density on cell membrane & $1-2^{1}$ & $\mathrm{number}$ of electron $/ \mathrm{nm}^{2}$ \\
$\gamma$ & Surface tension & $0.01-0.1^{14}$ & $\mathrm{pN} / \mathrm{nm}$ \\
$k_{1}$ & Spring constant & $\mathrm{Pa}$ \\
$k_{2}$ & Spring constant & $4-223^{\mathrm{b}}$ & $\mathrm{Pa}$ \\
$\mu$ & Viscosity & $2-175^{9}$ & $\mathrm{~Pa} \cdot \mathrm{S}$ \\
\hline
\end{tabular}

Sato et al. ${ }^{34,33}$, Lim et al. ${ }^{26}$.

aYuan et al. ${ }^{45}$.

${ }^{\text {b }}$ Boal ${ }^{5}$.

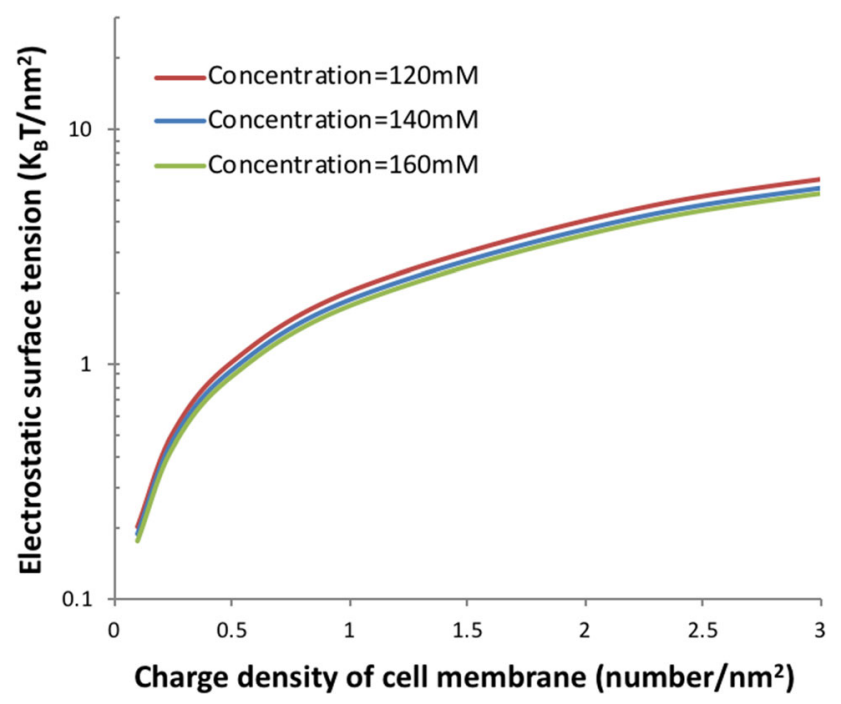

FIGURE 2. Effects of charge density of cell membrane and salt concentration on electrostatic surface tension of cell membrane.

can be neglected when compared to the total resistance, $1 / \mathrm{P}$, which is in the order of $10^{8} \mathrm{~s} / \mathrm{cm}$.

\section{Model Predictions}

As shown in Eqs. 1-4, in addition to the size of the NPs and the charge carried by the NPs, the bending rigidity, surface tension and the viscoelastic properties of the cell membrane, the charge carried by the cell membrane, as well as the salt concentrations in the surrounding solution, all of which contribute to the transcellular transport of the NP across the in vitro BBB. Therefore, the following sections show how each factor affects the transcellular transport of the NPs.
Table 2 summarizes the values or the range of values for these parameters determined from the experiments.

Effects of Salt Concentration in the Surrounding Solution and Charge Density of EC Membrane on Electrostatic Surface Tension

One factor affects the cell membrane deformation is the effective surface tension of the cell membrane, $\gamma_{\text {eff }}=\gamma-\gamma_{\text {ele }}$, of which the electrostatic surface tension $\gamma_{\text {ele }}$ contributes significantly. $\gamma$ is mechanical surface tension of the cell membrane. $\gamma_{\text {ele }}=\pi l_{\mathrm{B}} \sigma^{2} / \kappa$. Here $1_{\mathrm{B}}$ is the Bjerrum length, which is a constant. $1 / \kappa=$ is the Debye- Hückle length. $\kappa=\sqrt{8 \pi l_{\mathrm{B}} \mathrm{C}}$, here $\mathrm{C}$ is the salt concentration in the surrounding solution. The salt concentration $\mathrm{C}$ changes from 120 to $160 \mathrm{mM}$ in the blood plasma or in the culture medium used in the in vitro $\mathrm{BBB}$ model. $\sigma$ is the charge density of the cell membrane which ranges from 0 to 3 (number of electrons $/ \mathrm{nm}^{2}$ ) for the EC membrane in vivo and in vitro under physiological and pathological conditions. ${ }^{44,45}$ Figure 2 demonstrates how the electrostatic surface tension $\gamma_{\text {ele }}$ of the EC membrane is affected by the charge density $\sigma$ and salt concentration $C$. Although $\gamma_{\text {ele }}$ increases greatly with $\sigma$ but only has very minor changes with $\mathrm{C}$ of our solutions. In fact, this electrostatic surface tension $\gamma_{\text {ele }}$ of the cell membrane would bend the cell membrane to enable the endocytosis of a NP.

Effects of Bending Rigidity, Surface Tension and Charge Density of EC Membrane on Penetration Process of a Neutral NP

As defined in Eqs. 19-22, the BBB permeability $(P)$ to a neutral NP depends on its diameter $2 \mathrm{R}$, and the wrapping or penetrating time $t^{*}$ during endocytosis/ 
(a)

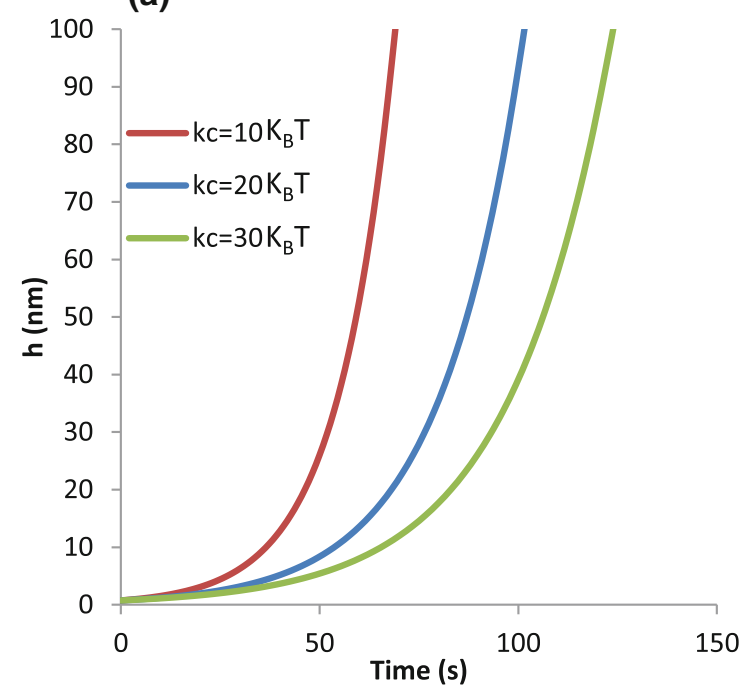

(b)

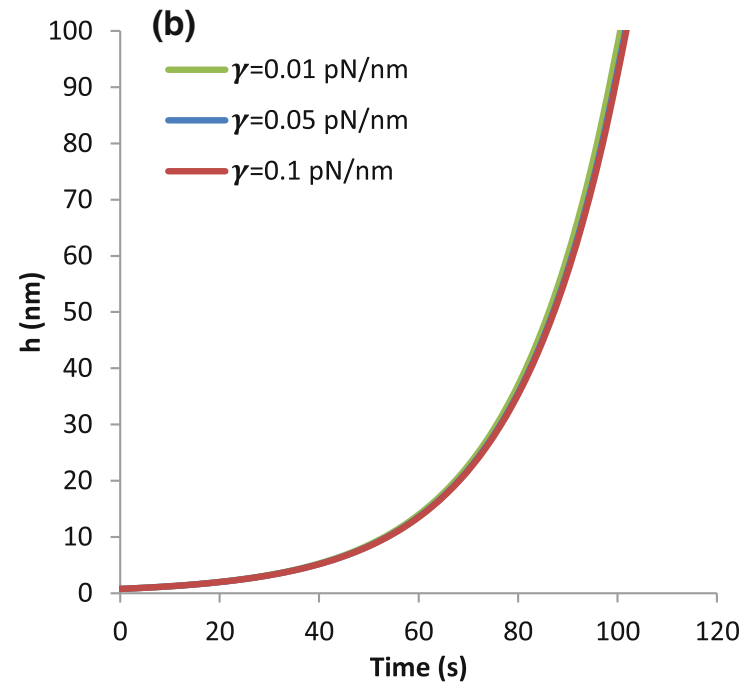

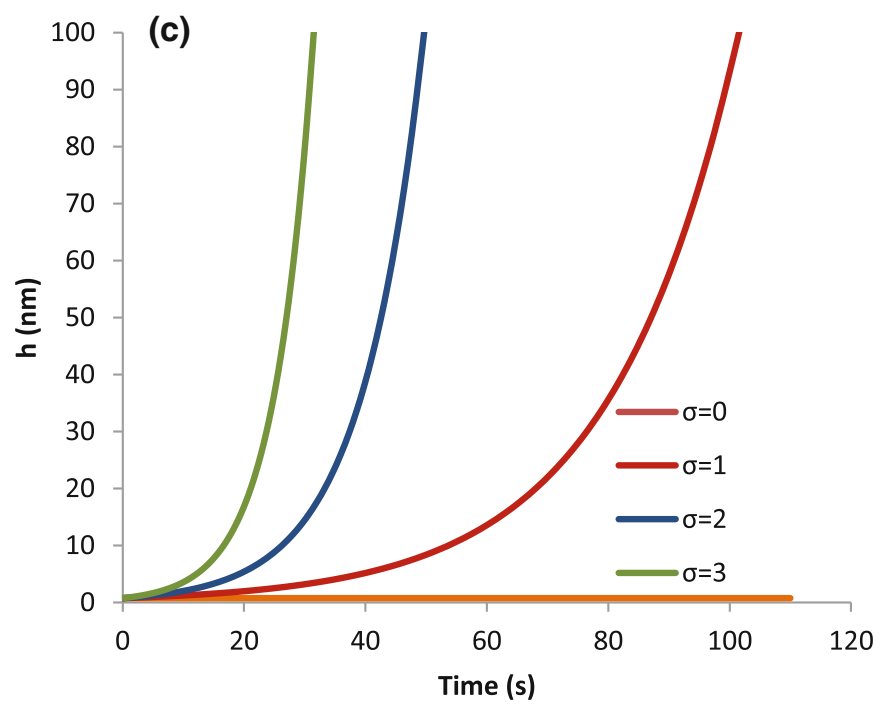

FIGURE 3. Effects of physical properties of cell membrane on penetration process of a neutral NP. Bending rigidity $k_{\mathrm{c}}$ (a), surface tension $\gamma(\mathrm{b})$, and charge density $\sigma$ (c) of cell membrane. Diameter of the NP is $100 \mathrm{~nm}$. The unit of $\sigma$ is $1 / \mathrm{nm}^{2}$.

exocytosis since the diffusion resistance in the EC cytoplasm is negligible as compared with the measured data. Figure 3 demonstrates the effects of bending rigidity $\mathrm{k}_{\mathrm{c}}$, surface tension $\gamma$ and charge density $\sigma$ of the EC membrane on the penetration process of a neutral $\mathrm{NP}$ with diameter $2 R=100 \mathrm{~nm}$. In Fig. 3, we fixed the viscoelastic properties of the EC membrane to the medium values from the experiments (Table 2): $k_{1}=k_{2}=100 \mathrm{~Pa}, \mu=6000 \mathrm{PaS}$. Figure 3a shows the effect of the bending rigidity $k_{\mathrm{c}}$ on the NP penetration, the higher the $k_{\mathrm{c}}$, the harder the penetration. When $\gamma=0.05 \mathrm{pN} / \mathrm{nm}, \sigma=1 / \mathrm{nm}^{2}$, the medium values from the measurements, if $\mathrm{k}_{\mathrm{c}}$ increases from 10 to $20 K_{\mathrm{B}} T$, by twofold, the wrapping time increases from 69 to $101 \mathrm{~s}$, by $\sim 1.5$-fold; when $k_{\mathrm{c}}$ further increases from 20 to $30 K_{\mathrm{B}} T$, the wrapping time increases by $\sim 1.23$-fold. While $k_{\mathrm{c}}$ significantly affects the penetration process, the mechanical surface tension $\gamma$ seems to have a negligible effect in the measured range of $0.01-$ $0.1 \mathrm{pN} / \mathrm{nm}$ for the EC membrane (Fig. 3b). In Fig. 3b, $k_{\mathrm{c}}=20 \mathrm{~K}_{\mathrm{B}} \mathrm{T}$ and $\sigma=1 / \mathrm{nm}^{2}$. Because the negative charge carried by the EC membrane $\sigma$ greatly affects the electrostatic surface tension (Fig. 2), it should contribute significantly to the penetration of a NP. When $\sigma$ increases from 1 to $3 / \mathrm{nm}^{2}$, the wrapping time decreases from 102 to $32 \mathrm{~s}$ (Fig. 3c). In Fig. 3c, $k_{\mathrm{c}}=20 K_{\mathrm{B}} T, \gamma=0.05 \mathrm{pN} / \mathrm{nm}$. In contrast, if EC carries no charge, $\sigma=0$, it is impossible for a neutral NP to penetrate across the EC membrane with aforementioned physical and mechanical properties. 
(a)

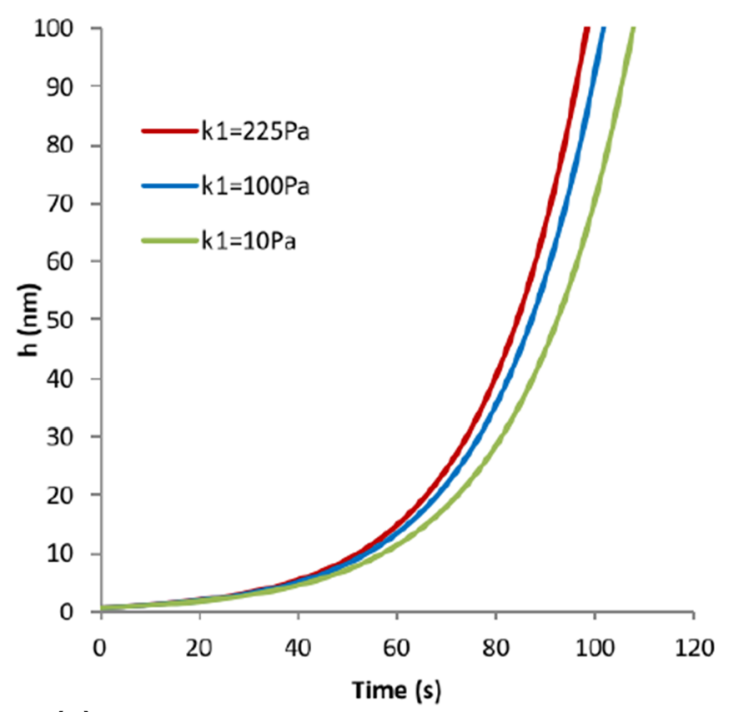

(c)

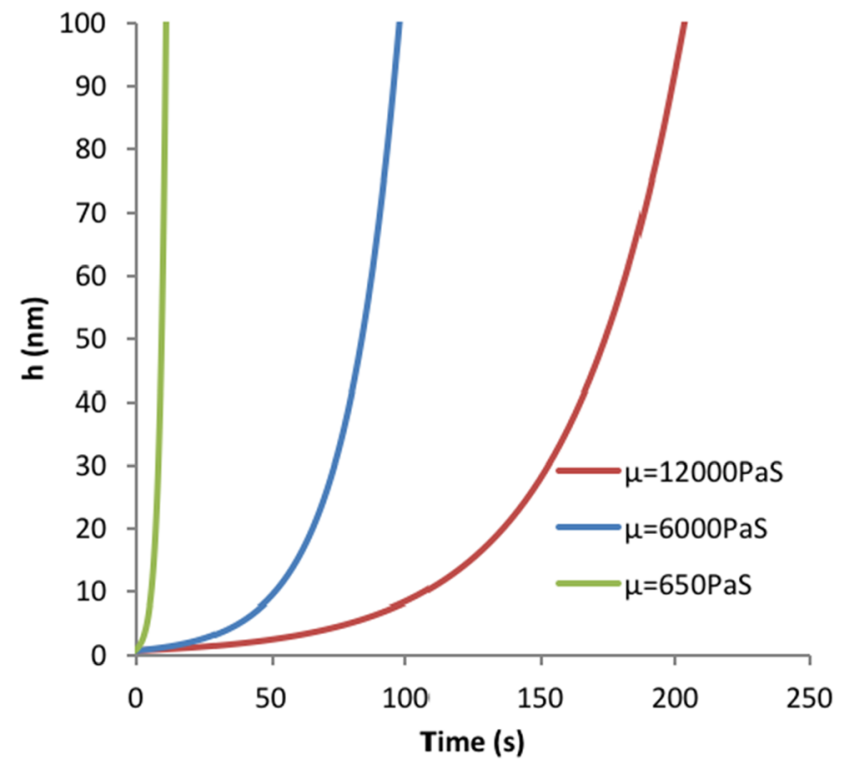

(b)

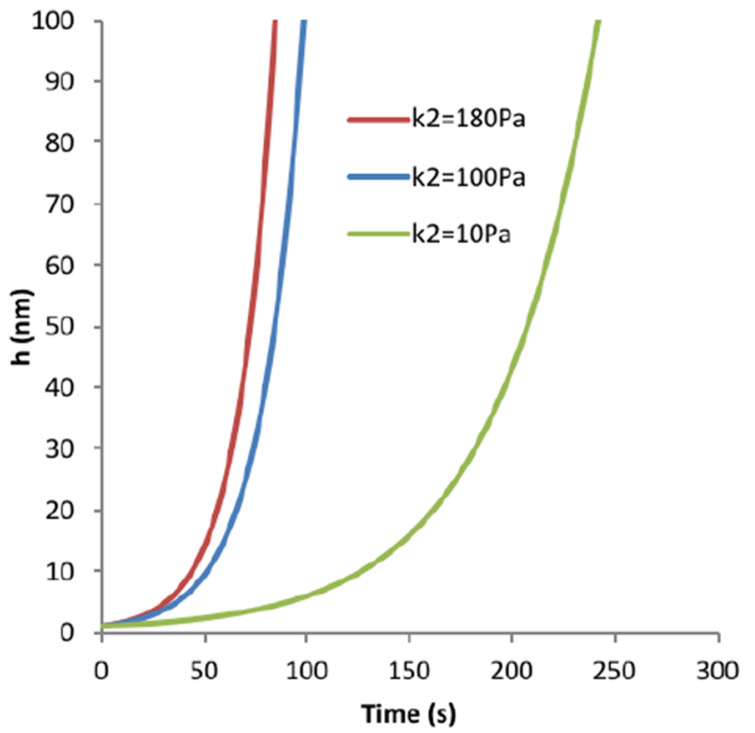

FIGURE 4. Effects of viscoelastic properties of cell membrane on penetration process of a neutral NP. Spring constants $k_{1}(a)$ and $k_{2}(b)$, and viscosity $\mu$ (c) in a standard linear solid model for cell membrane. Diameter of the NP is $100 \mathrm{~nm}$.

Effects of Viscoelastic Properties of EC membrane on Penetration Process of a Neutral NP

Since we used a standard linear solid (SLS) model to describe the viscoelastic properties of the EC membrane, we investigated how the viscoelastic parameters affect the penetration process of a neutral NP. Figure 4 demonstrates these effects. In Fig. 4, we chose $k_{\mathrm{c}}=20$ $K_{\mathrm{B}} T, \gamma=0.05 \mathrm{pN} / \mathrm{nm}, \sigma=1 / \mathrm{nm}^{2}$, the medium values from the experiments for the physical properties of the $\mathrm{EC}$ membrane, and NP diameter $=100 \mathrm{~nm}$. Figure $4 \mathrm{a}$ shows that the spring constant $\mathrm{k}_{1}$ in the SLS model seems not to significantly affect the NP penetration process while Fig. $4 \mathrm{~b}$ indicates that the penetration is sensitive to another spring constant $k_{2}$, which is in series with the dashpot $(\mu)$ of the SLS model. When $k_{2}$ changes from 10 to $100 \mathrm{~Pa}$, the penetrating time decreases from 241 to $98 \mathrm{~s}$. Figure $3 \mathrm{c}$ demonstrates that the penetration is also sensitive to viscosity $\mu$ (dashpot) of the EC membrane. When $\mu$ decreases from 12,000 to $6000 \mathrm{PaS}$, the penetrating time decreases from 203 to $98 \mathrm{~s}$; while $\mu$ decreases from 6000 to $650 \mathrm{PaS}$, the penetrating time further decreases from 98 to $11 \mathrm{~s}$. 


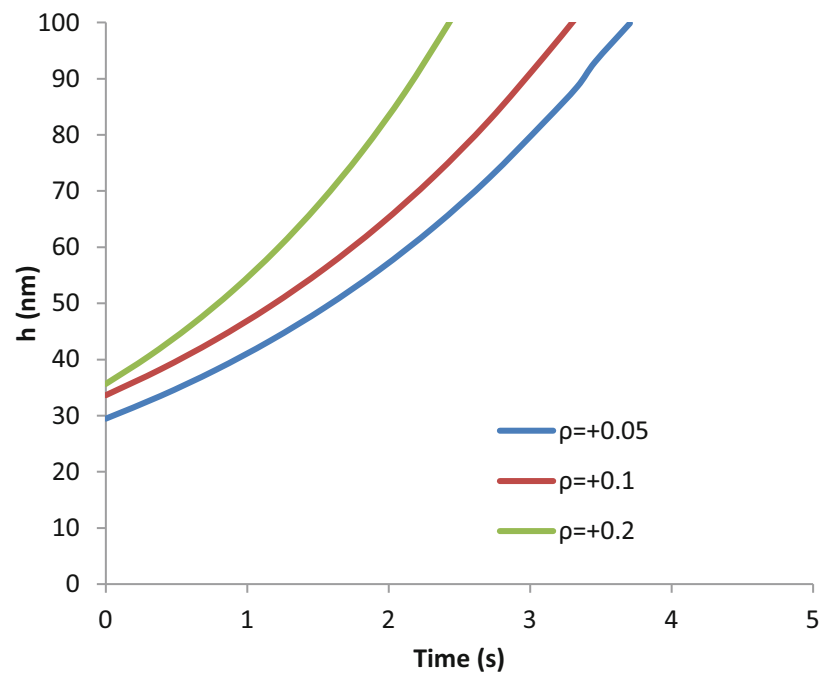

FIGURE 5. Effect of charge density of the NP on the penetration process of positively charged NPs. The unit of charge density of NPs $\rho$ is $1 / \mathrm{nm}^{2}$. Diameter of the NP is $100 \mathrm{~nm}$.

\section{Effect of Charge Density of NPs on the Penetration} Process of a Positively Charged NP

When a NP carries opposite charge of the cell membrane, the NP is attracted to the cell membrane by the electrostatic force to enhance its penetration. Since the EC membrane carries negative charge, we investigated the effect of charge density of a positively charged NP on its penetration process. The diameter of the NP is $100 \mathrm{~nm}$. Other parameters used for physical and viscoelastic properties of the EC membrane were fixed as the medium values from the experiments, which are $k_{\mathrm{c}}=20 K_{\mathrm{B}} T, \gamma=0.05 \mathrm{pN} / \mathrm{nm}, \sigma=1 / \mathrm{nm}^{2}$, and $k_{1}=k_{2}=100 \mathrm{~Pa}, \mu=6000 \mathrm{PaS}$. Figure 5 demonstrates that there is an initial penetration depth at the EC membrane depending on the charge density $\rho$ carried by the NP, the larger the $\rho$, the deeper the initial penetration. But the variation is within $20 \%$ when $\rho$ increases by fourfolds, from 0.05 to $0.2 / \mathrm{nm}^{2}$. For this change in $\rho$, the wrapping time decreases from 3.7 to $2.4 \mathrm{~s}$, about 1.54 folds.

Effect of NP Size on the Penetration Process of Neutral and Charged NPS

Figure 6 demonstrates how the size of the NP affect its penetration process. The values for the physical and viscoelastic property parameters for the EC membrane are the same as those used in Fig. 5. The diameter of the NP was chosen as 20,50,100 nm, the typical size used in NP drug delivery and also used in our in vitro experiment. Figure 6a shows those for neutral NPs. Although the penetrating time $t^{*}$ varies largely for various sized NPs, from 42.4, 85.7, to $143.2 \mathrm{~s}$, the (a)
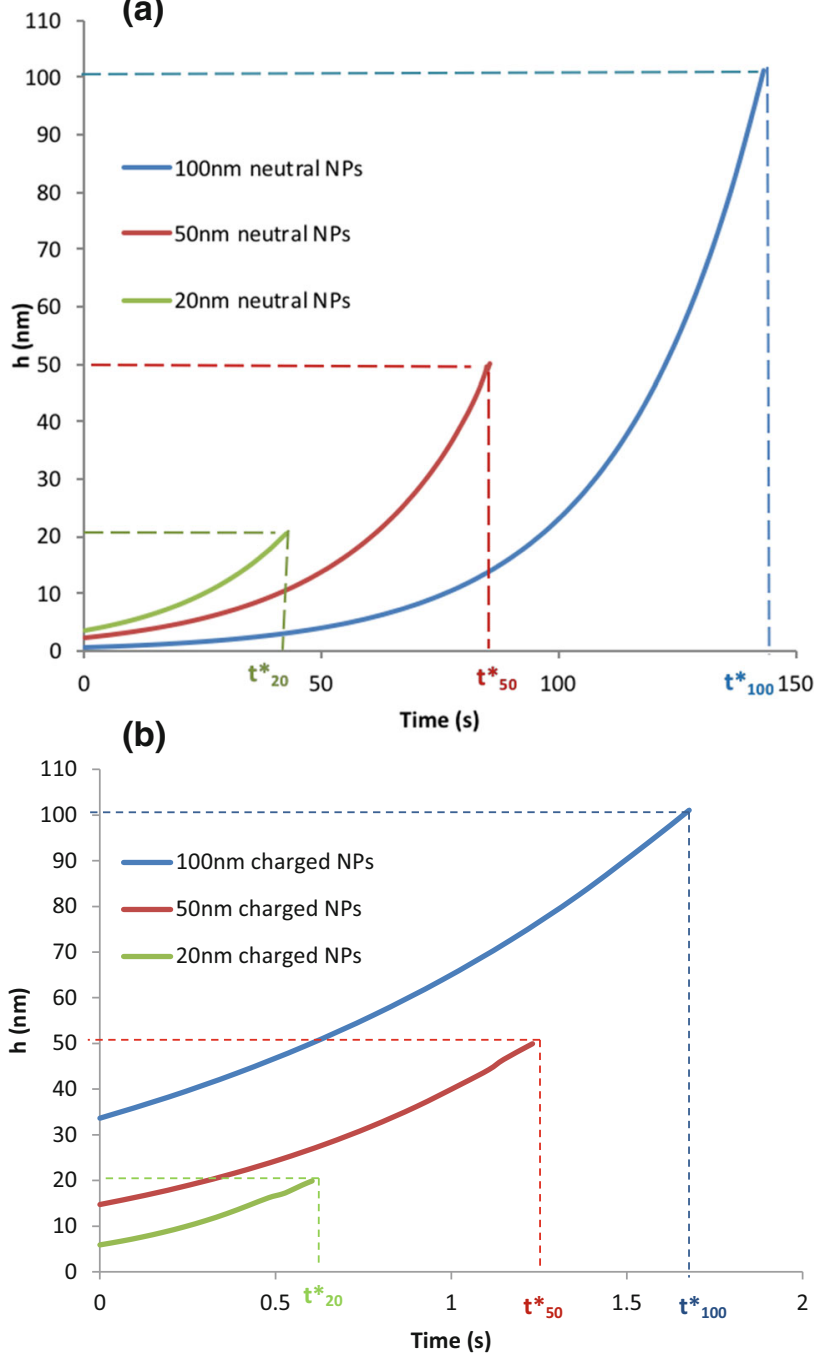

FIGURE 6. Effects of the size of NPs on the penetration process. For neutral NPs (a) and positively charged NPs with charge density $\rho$ of $0.1 / \mathrm{nm}^{2}$ (b).

permeability of an EC membrane, $2 R / t^{*}$, is $0.47,0.58$ and $0.69 \mathrm{~nm} / \mathrm{s}$, quite similar for the NP with diameter 20, 50 and $100 \mathrm{~nm}$, respectively. Figure $6 \mathrm{~b}$ is for the positively charged NPs with the same charge density $\rho=0.1 / \mathrm{nm}^{2}$. We can see that the penetrating time is greatly reduced for the charged NP compared to the same sized neutral NP, from $0.6,1.2$, to $1.7 \mathrm{~s}$ for the $\mathrm{NP}$ with diameter 20,50, and $100 \mathrm{~nm}$. As a result, the permeability of the EC membrane to the charged NP is 33.3, 41.7 and $58.8 \mathrm{~nm} / \mathrm{s}$, correspondingly.

Comparison of Model Prediction with Measured Permeability of In Vitro BBB to Neutral and Charged NPS

Figure 7 demonstrates the comparison between the in vitro $\mathrm{BBB}$ (bEnd3 monolayer) permeability $(\mathrm{P})$ predicted by the transcellular model and that measured in the experiments. Figure 7a shows the results for the 


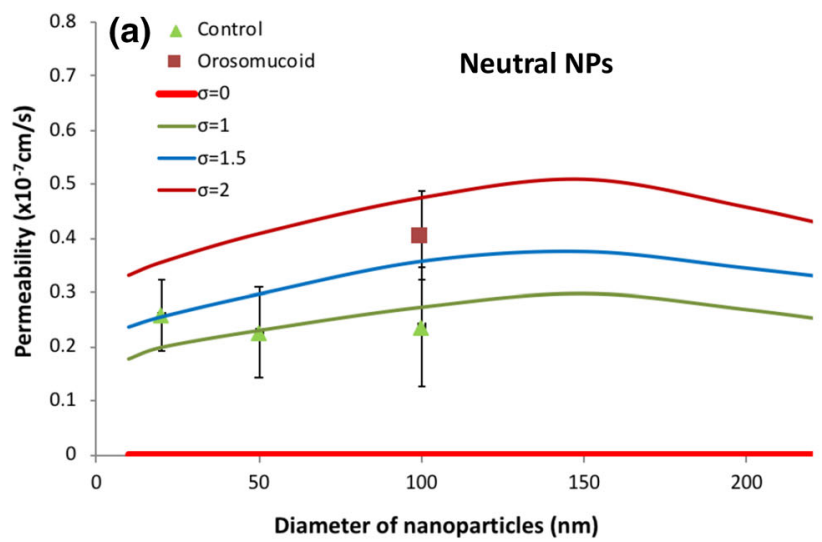

(b)

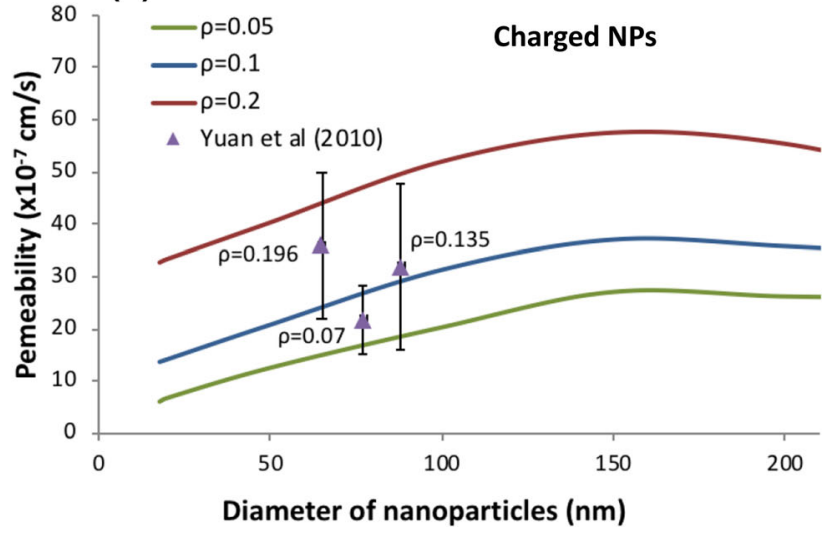

FIGURE 7. Comparison of the BBB permeability predicted by the transcellular model and that measured in the experiments. For neutral NPs (a) and positively charged NPs (b). Lines are model predictions and symbols are measured data. The unit for charge density of the cell membrane $\sigma$ and that for the charge density of NPs $\rho$ are $1 / \mathrm{nm}^{2}$.

$P$ to neutral NPs as a function of charge density $\sigma$ of the EC membrane and the size of NPs. Other parameters of the EC membrane are the same as those in Fig. 6 except $k_{1}=110 \mathrm{~Pa}$ and $\mathrm{k}_{2}=60 \mathrm{~Pa}$. Lines in Fig. 7a are the model predictions for changing charge density $\sigma$ of the EC membrane, from 0 to $2 / \mathrm{nm}^{2}$. When there is no charge at the EC membrane, $\sigma=0$, no NPs can penetrate the EC monolayer, $P=0$. The surface charge of the bEnd3 monolayer is crucial in neutral NP transport across the monolayer. As long as the surface charge is large enough, there is always a chance for a neutral NP in this size range passing through the monolayer. The larger the surface charge density, the higher the NP permeability. Another observation is that the permeability of the monolayer is almost insensitive to the size of the NP in this size range although there is a slight increase when NP diameter is from 20 to $100 \mathrm{~nm}$. However, the optimal size of the $\mathrm{NP}$ for this type of EC monolayer is around $150 \mathrm{~nm}$ to achieve the highest permeability. Comparing the model predictions with the measured P of neutral NPs (green colored symbols, from Table 1), we can see that for the best fit to the measured $P$, the charge density $\sigma$ of the EC membrane would be $\sim 1 / \mathrm{nm}^{2}$. This represents a $100-150 \mathrm{~nm}$ thick surface glycocalyx with volume charge density $\sim 20 \mathrm{mEq} / \mathrm{L}$, which is indeed in the range of what estimated for the bEnd3 monolayer under control conditions by Ref. 44. Furthermore, after the bEnd 3 monolayer was treated by $1 \mathrm{mg} / \mathrm{mL}$ orosomucoid, a plasma glycoprotein, the permeability to a neutral NP of $100 \mathrm{~nm}$ increases $\sim 1.8$-fold. Previous studies showed that orosomucoid modulates the charge of the intact BBB as well as bEnd3 monolayer. Orosomucoid of $1 \mathrm{mg} / \mathrm{mL}$ increases charge density of bEnd 3 monolayer to $\sim$ twofold of its control value. ${ }^{44}$ The predictions at $\sigma=1.5$ and $2 / \mathrm{nm}^{2}$ indeed enclose the increased $\mathrm{P}$ by orosomucoid (Fig. 7a).

Figure $7 \mathrm{~b}$ compares the model predictions and measured $P^{44}$ of the bEnd 3 monolayer to the positively charged NPs. The charge density of the EC membrane $\sigma$ was chosen as $1.0 / \mathrm{nm}^{2}$, the control value determined in Ref. 44 for bEnd3 monolayer. Lines are model predictions when the charge density $\rho$ of the NP $=$ $0.05,0.1$ and $0.2 / \mathrm{nm}^{2}$. The symbols are measured permeability data for the three sized NPs with different charge density $0.07,0.135$ and $0.196 / \mathrm{nm}^{2}$. Figure $7 \mathrm{~b}$ shows that our model predictions are in very good agreement with the measured data. We can see from Fig. $7 \mathrm{~b}$ that the charge carried by the NP greatly enhances its transcellular permeability by an order of two magnitudes compared to their neutral counterparts (Fig. 7a). The same as for the neutral NP, the highest permeability of a charged NP occurs when the NP is around $150 \mathrm{~nm}$ for this type of EC monolayer; also when the cell surface charge is zero, no charged NP can cross the monolayer no matter how much charge it carries (not shown).

\section{DISCUSSION}

In this study, we quantified the permeability of an in vitro $\mathrm{BBB}$ (bEnd3 monolayer) to neutral NPs with the typical sizes used in drug delivery. This size range $(20-100 \mathrm{~nm})$ is much larger than the inter-endothelial cleft and thus the transport of these NPs across the BBB is through transcytosis or transcellular pathway. In order to understand the transcellular mechanism by which the NPs cross the BBB, we developed a transcell membrane model based on a theory for the mechanical and electrostatic energy of a charged membrane interacting with a neutral and oppositely charged sphere in a salt solution, as well as a standard linear solid model for the viscoelastic property of the cell membrane. We simplified the transcellular transport of a NP into an endocysis process, diffusion 
across the cytoplasm and an exocytosis process. We also assumed that it takes the same amount of time for a NP for the endocytosis and for the exocytosis.

Different from the receptor-mediated transcytosis, which needs specific receptors such as the insulin receptor, trasnferrin receptor and LDL (low-density lipoprotein) receptor to cross the $\mathrm{BBB},{ }^{14,31}$ the surface charge and mechanical property-mediated transcytosis is nonspecific and driven by electrochemical potential gradients. ${ }^{13}$ In general, most biomembranes are negatively charged due to their compositions and the surrounding salt solutions. ${ }^{18,40}$ This indicates a new direction for designing NPs for brain drug delivery through the BBB. Specifically, the EC forming the BBB carries negative charge not only at the luminal side, due to surface glycocalyx, but also at the abluminal side, due to extracellular matrix in the basement membrane..$^{10,11,44}$ The charge density is comparable at the luminal and abluminal sides under control conditions, ${ }^{23}$ which justified the assumption that it takes the same amount of time for a NP for the endocytosis and for the exocytosis.

On the basis of the estimated charge density of the in vitro $\mathrm{BBB}$ (bEnd 3 monolayer ${ }^{44}$ and the measured mechanical properties of the ECs, ${ }^{26,34,48}$ our transcellular model indicates that the surface charge carried by the EC plays an essential role in NP transcytosis for the NP with diameter of 20-200 $\mathrm{nm}$. If the EC carries no charge, no NPs can cross the in vitro BBB regardless of whether the NPs carry charge or not. In contrast, if the EC carries negative charge, there is an opportunity for a neutral NP to go transcytosis even though the rate (or the pemeability) may be very small if the charge density is small. For the same sized NP, if it carries positive charge, though as small as $0.05 / \mathrm{nm}^{2}$, its permeability would be enhanced by $\sim 100$-fold under normal conditions, compared to its neutral counterpart. This amount of the NP charge density is safe and biocompatible. ${ }^{17}$ For both neutral and positively charged NPs, our model also predicted that the optimal size would be $\sim 150 \mathrm{~nm}$ to achieve the highest BBB permeability in the physiological conditions. This optimal size varies for different type of cells and under different conditions. In addition to modulating the charge and the size of the NP, we can modulate the charge density of the BBB by applying orosomucoid, a plasma protein, or by adding negatively charged glycocalyx components, glycosaminoglycans, at their safe dose levels. Furthermore, we can modulate the mechanical properties of EC membrane as well as those of the NPs. ${ }^{21}$

In this study, we assumed that as long as a NP is completely engulfed by the cell membrane, it accomplishes the endocytosis. For this, we assumed that the time it takes for a NP to overcome the viscous friction of the cell membrane is much longer than that for a NP to break the cell membrane and that for the cell membrane to go back to its initial flat shape. Yuan et al $^{47}$ showed that the recovering time of the cell membrane of a Hela cell from a deformed shape to its original one is about $1 \mathrm{~ms}$. If using this time scale for the recovery of the cell membrane in our study, it is negligible compared to the NP penetrating (engulfing) time, which is several $10 \mathrm{~s}$ to over $100 \mathrm{~s}$. We were unable to justify that the time for a NP to break the cell membrane is much shorter than the engulfing time and that a NP can be in the cytoplasm before it is completely wrapped by the cell membrane. We also did not consider the discontinuity at the torn edge of the cell membrane. The only validation for these assumptions is that our current model predictions match the measured data. Future improvement can be made by adding these factors.

Although the above explanation may tell how NPs go through the endo/exocytosis across the EC membrane, how they transport inside EC cytoplasm is unclear. The intracellular transport of these NPs may be mediated by the vesicular system, e.g., by caveolae or macropinocytotic vesicles. ${ }^{27,30,31}$ In the current model, we simplied the intracellular transport of the NP to a pure diffusion. Compared to the endo/exocytosis, the time taken by the diffusion is negligible. Further investigation will be conducted for the details in the NP intracellular transport.

In summary, we developed a transcellular model for describing the NP endo/exocytosis induced by the surface charge and mechanical properties of the EC membrane, the salt concentration of the surrounding solution, as well as the size and surface charge of the NP. The predictions from the model can explain the measured permeability data of an in vitro $\mathrm{BBB}$ to neutral and charged NPs. This model can be further used to design optimal systemic brain drug delivery strategies by predicting the optimal size and charge for a drug-loaded NP for the EC membrane with the specific surface charge and mechanical properties. We can also use agents to modulate the EC surface charge and mechanical properties to achieve optimal delivery effects. Since pathogens such as COVID-19 viruses have the size of 50-200 nm, similar to the size of the NPs, guided by our model, we can change the physical and mechanical properties of the cell membrane, as well as the salt concentration of the surrounding solution to inhibit their entry into the cell.

\section{ACKNOWLEDGMENTS}

We would like to thank Dr. John Tarbell's lab at the City College of New York to allow us to use the automated fluorometer system for measuring the per- 
meability to neutral NPs. We also would like to thank the funding support from NSF CBET-0754158, NIH CA 153325-01 and 1UH3TR002151-01.

\section{CONFLICT OF INTEREST}

The authors declare that they have no conflict of interest.

\section{REFERENCES}

${ }^{1}$ Abbott, N. J. Blood-brain barrier structure and function and the challenges for CNS drug delivery. J. Inherit. Metab. Dis. 36:437-449, 2013.

${ }^{2}$ Allt, G., and J. G. Lawrenson. Is the pial microvessel a good model for blood-brain barrier studies? Brain Res. Brain Res. Rev. 24:67-76, 1997.

${ }^{3}$ Ando, Y., H. Okada, G. Takemura, K. Suzuki, C. Takada, H. Tomita, et al. Brain-specific ultrastructure of capillary endothelial glycocalyx and its possible contribution for blood brain barrier. Sci. Rep. 8:17523, 2018.

${ }^{4}$ Bannunah, A. M., D. Vllasaliu, J. Lord, and S. Stolnik. Mechanisms of nanoparticle internalization and transport across an intestinal epithelial cell model: effect of size and surface charge. Mol. Pharm. 11:4363-4373, 2014.

${ }^{5}$ Boal, D. H. Mechanics of the Cell (2nd ed.). Cambridge: Cambridge University Press, 2012.

${ }^{6}$ Brigger, I., J. Morizet, G. Aubert, H. Chacun, M. J. Terrier-Lacombe, P. Couvreur, et al. Poly(ethylene glycol)coated hexadecylcyanoacrylate nanospheres display a combined effect for brain tumor targeting. J. Pharmacol. Exp. Ther. 303:928-936, 2002.

${ }^{7}$ Cancel, L. M., A. Fitting, and J. M. Tarbell. In vitro study of LDL transport under pressurized (convective) conditions. Am. J. Physiol. Heart Circ Physiol. 293:H126-H132, 2007.

${ }^{8}$ Cancel, L. M., and J. M. Tarbell. The role of apoptosis in LDL transport through cultured endothelial cell monolayers. Atherosclerosis. 208:335-341, 2010.

${ }^{9}$ DeAngelis, L. M. Brain tumors. N. Engl. J. Med. 344:114123, 2001.

${ }^{10}$ Fan, J., B. Cai, M. Zeng, Y. Hao, F. G. Giancotti, and B. $\mathrm{M}$. Fu. Integrin beta4 signaling promotes mammary tumor cell adhesion to brain microvascular endothelium by inducing ErbB2-mediated secretion of VEGF. Ann. Biomed. Eng. 39:2223-2241, 2011.

${ }^{11}$ Fan, J., and B. M. Fu. Quantification of malignant breast cancer cell MDA-MB-231 transmigration across brain and lung microvascular endothelium. Ann. Biomed. Eng. 44:2189-2201, 2016.

${ }^{12}$ Feng, S. S., L. Mu, K. Y. Win, and G. Huang. Nanoparticles of biodegradable polymers for clinical administration of paclitaxel. Curr. Med. Chem. 11:413-424, 2004.

${ }^{13}$ Fleck, C. C., and R. R. Netz. Electrostatic colloid-membrane binding. Europhys. Lett. 67:314-320, 2004.

${ }^{14} \mathrm{Fu}$, B. M. Transport across the blood-brain barrier. $A d v$. Exp. Med. Biol. 1097:235-259, 2018.

${ }^{15} \mathrm{Fu}$, B. M., B. Chen, and W. Chen. An electrodiffusion model for effects of surface glycocalyx layer on microvessel permeability. Am. J. Physiol. Heart Circ Physiol. 284:H1240-H1250, 2003.

${ }^{16} \mathrm{Gao}, \mathrm{H}$., W. Shi, and L. B. Freund. Mechanics of receptormediated endocytosis. Proc. Natl. Acad. Sci. USA. 102:9469-9474, 2005.

${ }^{17}$ Gil, E. S., J. Li, H. Xiao, and T. L. Lowe. Quaternary ammonium beta-cyclodextrin nanoparticles for enhancing doxorubicin permeability across the in vitro blood-brain barrier. Biomacromolecules. 10:505-516, 2009.

${ }^{18} \mathrm{Gm}$, C. The Cell: A Molecular Approach (2nd ed.). Sunderland (MA): Sinauer Associates, 2000.

${ }^{19}$ Harush-Frenkel, O., E. Rozentur, S. Benita, and Y. Altschuler. Surface charge of nanoparticles determines their endocytic and transcytotic pathway in polarized MDCK cells. Biomacromolecules. 9:435-443, 2008.

${ }^{20}$ Hawkins, B. T., and T. P. Davis. The blood-brain barrier/ neurovascular unit in health and disease. Pharmacol. Rev. 57:173-185, 2005.

${ }^{21}$ Hui, Y., X. Yi, F. Hou, D. Wibowo, F. Zhang, D. Zhao, et al. Role of nanoparticle mechanical properties in cancer drug delivery. ACS Nano. 13:7410-7424, 2019.

${ }^{22}$ Jamali, Y., M. Azimi, and M. R. Mofrad. A sub-cellular viscoelastic model for cell population mechanics. PLoS ONE 5(8):e12097, 2010.

${ }^{23} \mathrm{Li}, \mathrm{G}$., and B. M. Fu. An electrodiffusion model for the blood-brain barrier permeability to charged molecules. $J$. Biomech. Eng. 133:021002, 2011.

${ }^{24}$ Li, G., M. J. Simon, L. M. Cancel, Z. D. Shi, X. Ji, J. M. Tarbell, et al. Permeability of endothelial and astrocyte cocultures: in vitro blood-brain barrier models for drug delivery studies. Ann. Biomed. Eng. 38:2499-2511, 2010.

${ }^{25} \mathrm{Li}, \mathrm{G}$., W. Yuan, and B. M. Fu. A model for the bloodbrain barrier permeability to water and small solutes. $J$. Biomech. 43:2133-2140, 2010.

${ }^{26}$ Lim, C. T., E. H. Zhou, and S. T. Quek. Mechanical models for living cells-a review. J. Biomech. 39:195-216, 2006.

${ }^{27}$ Mayor, S., R. G. Parton, and J. G. Donaldson. Clathrinindependent pathways of endocytosis. Cold Spring Harbor Perspect. Biol. 6(6):93e112, 2014.

${ }^{28}$ Olivier, J. C. Drug transport to brain with targeted nanoparticles. NeuroRx. 2:108-119, 2005.

${ }^{29}$ Pardridge, W. M. Molecular biology of the blood-brain barrier. Mol. Biotechnol. 30:57-70, 2005.

${ }^{30}$ Parkar, N. S., B. S. Akpa, L. C. Nitsche, L. E. Wedgewood, A. T. Place, M. S. Sverdlov, et al. Vesicle formation and endocytosis: function, machinery, mechanisms, and modeling. Antioxid. Redox Signal. 11:1301-1312, 2009.

${ }^{31}$ Pulgar, V. M. Transcytosis to cross the blood brain barrier, new advancements and challenges. Front. Neurosci. $12: 1019,2018$

${ }^{32}$ Saraiva, C., C. Praca, R. Ferreira, T. Santos, L. Ferreira, and L. Bernardino. Nanoparticle-mediated brain drug delivery: Overcoming blood-brain barrier to treat neurodegenerative diseases. J. Control Rel. 235:34-47, 2016.

${ }^{33}$ Sato, M., N. Ohshima, and R. M. Nerem. Viscoelastic properties of cultured porcine aortic endothelial cells exposed to shear stress. J. Biomech. 29:461-467, 1996.

${ }^{34}$ Sato, M., D. P. Theret, L. T. Wheeler, N. Ohshima, and R. M. Nerem. Application of the micropipette technique to the measurement of cultured porcine aortic endothelial cell viscoelastic properties. J. Biomech. Eng. 112:263-268, 1990.

${ }^{35}$ Shi, L., P. Palacio-Mancheno, J. Badami, D. W. Shin, M. Zeng, L. Cardoso, et al. Quantification of transient increase of the blood-brain barrier permeability to macromolecules 
by optimized focused ultrasound combined with microbubbles. Int. J. Nanomed. 9:4437-4448, 2014.

${ }^{36}$ Shi, L., M. Zeng, Y. Sun, and B. M. Fu. Quantification of blood-brain barrier solute permeability and brain transport by multiphoton microscopy. J. Biomech. Eng. 136:031005, 2014.

${ }^{37}$ Singh, A., G. Garg, and P. Sharma. Nanospheres: A novel approach for targeted drug delivery system. Int. J. Pharm. Sci. Rev. Res. 5(3):15, 2010.

${ }^{38}$ Smith, K. R., and R. T. Borchardt. Permeability and mechanism of albumin, cationized albumin, and glycosylated albumin transcellular transport across monolayers of cultured bovine brain capillary endothelial cells. Pharm. Res. 6:466-473, 1989.

${ }^{39}$ Teleanu, D. M., C. Chircov, A. M. Grumezescu, A. Volceanov, and R. I. Teleanu. Blood-brain delivery methods using nanotechnology. Pharmaceutics 10(4):269, 2018.

${ }^{40}$ Winterhalter, M., and W. Helfrich. Effect of surface-charge on the curvature elasticity of membranes. J. Phys. Chem.Us. 92:6865-6867, 1988.

${ }^{41}$ Yacobi, N. R., N. Malmstadt, F. Fazlollahi, L. DeMaio, R. Marchelletta, S. F. Hamm-Alvarez, et al. Mechanisms of alveolar epithelial translocation of a defined population of nanoparticles. Am. J. Respir. Cell Mol. Biol. 42:604-614, 2010.

${ }^{42}$ Yen, W. Y., B. Cai, M. Zeng, J. M. Tarbell, and B. M. Fu. Quantification of the endothelial surface glycocalyx on rat and mouse blood vessels. Microvasc. Res. 83:337-346, 2012.
${ }^{43}$ Yi, X., X. Shi, and H. Gao. A universal law for cell uptake of one-dimensional nanomaterials. Nano Lett. 14:10491055, 2014.

${ }^{44}$ Yuan, W., G. Li, E. S. Gil, T. L. Lowe, and B. M. Fu. Effect of surface charge of immortalized mouse cerebral endothelial cell monolayer on transport of charged solutes. Ann. Biomed. Eng. 38:1463-1472, 2010.

${ }^{45}$ Yuan, W., G. Li, M. Zeng, and B. M. Fu. Modulation of the blood-brain barrier permeability by plasma glycoprotein orosomucoid. Microvasc. Res. 80:148-157, 2010.

${ }^{46}$ Yuan, W., Y. Lv, M. Zeng, and B. M. Fu. Non-invasive measurement of solute permeability in cerebral microvessels of the rat. Microvasc. Res. 77:166-173, 2009.

${ }^{47}$ Yuan, F., C. Yang, and P. Zhong. Cell membrane deformation and bioeffects produced by tandem bubble-induced jetting flow. Proc. Natl. Acad. Sci. USA 112:E7039-E7047, 2015.

${ }^{48}$ Zhang, S., H. Gao, and G. Bao. Physical principles of nanoparticle cellular endocytosis. ACS Nano. 9:8655-8671, 2015.

${ }^{49}$ Zhong-can, O. Y., and W. Helfrich. Instability and deformation of a spherical vesicle by pressure. Phys. Rev. Lett. 59:2486-2488, 1987.

Publisher's Note Springer Nature remains neutral with regard to jurisdictional claims in published maps and institutional affiliations. 